This item was submitted to Loughborough's Research Repository by the author.

Items in Figshare are protected by copyright, with all rights reserved, unless otherwise indicated.

\title{
Borosilicate glass potentials for radiation damage simulations
}

PLEASE CITE THE PUBLISHED VERSION

http://dx.doi.org/10.1016/j.nimb.2014.12.024

\section{PUBLISHER}

(c) Elsevier

\section{VERSION}

AM (Accepted Manuscript)

\section{PUBLISHER STATEMENT}

This work is made available according to the conditions of the Creative Commons Attribution-NonCommercialNoDerivatives 4.0 International (CC BY-NC-ND 4.0) licence. Full details of this licence are available at: https://creativecommons.org/licenses/by-nc-nd/4.0/

\section{LICENCE}

CC BY-NC-ND 4.0

\section{REPOSITORY RECORD}

Jolley, Kenny, Roger Smith, and Kitheri Joseph. 2019. "Borosilicate Glass Potentials for Radiation Damage Simulations". figshare. https://hdl.handle.net/2134/16926. 


\title{
Borosilicate glass potentials for radiation damage simulations
}

\author{
Kenny Jolley ${ }^{\mathrm{a}, *}$, Roger Smith ${ }^{\mathrm{a}}$, Kitheri Joseph ${ }^{\mathrm{b}}$ \\ ${ }^{a}$ Mathematical Sciences, Loughborough University, Loughborough, LE11 3TU, UK. \\ ${ }^{b}$ Materials Chemistry Group, Indira Gandhi Research Centre, Kalpakkam, Tamil Nadu, \\ India
}

\begin{abstract}
Three borosilicate glass $\left(\mathrm{SiO}_{2}-\mathrm{B}_{2} \mathrm{O}_{3}\right)$ fixed charge potentials from the literature are compared $[1,2,3]$ and their suitability for use in simulations of radiation damage is assessed.

For a range of densities, we generate glass structures by quenching at $5 \times$ $10^{12} \mathrm{~K} / \mathrm{s}$ using constant volume Molecular Dynamics. In each case, the bond lengths, mean bond angles, bulk modulus, melting point and displacement energy thresholds are calculated and where possible compared to experimental data. Whereas the bond lengths and mean bond angles are reasonably well predicted, we find that the potentials predict melting temperatures, bulk moduli and densities that are higher than experimental data.

The displacement energy thresholds are generally lower than those for ionic crystalline materials but show a wider spread of values. However the barriers for atomic rearrangements after atoms have been displaced in the equilibrium structures are very high. This indicates that the radiation damage produced in the ballistic phase of a collision cascade is likely to persist for extended times scales in contrast to crystals where interstitials and vacancies can diffuse rapidly between successive radiation events.
\end{abstract}

\section{Introduction}

High-level nuclear Wastes (HLW) from spent nuclear power station fuels and decommissioned nuclear weapons must be safely removed from the environment. One leading method of achieving this, is long term storage by immobilisation in glass waste forms prior to permanent disposal in a geologically stable repository $[4,5]$. A primary concern with this method is that over long time-scales the radioactive material could leach out of the glass and contaminate the environment. It is for this reason we must understand how glass responds to radiation

\footnotetext{
* Corresponding author

Email addresses: kj14@alumni.le.ac.uk (Kenny Jolley), R.Smith@lboro.ac.uk (Roger Smith), joskit@gmail.com (Kitheri Joseph)
} 
damage over long time scales. In the early stages of waste encapsulation the material is heated due to the emission of electrons, however in this work it is the damage caused by $\alpha$ - decay that will be the main focus of the study.

Experiments on physical samples are limited to relatively short times (10 years) compared to geological time scales or are carried out at higher dose rates such as by using ion beams to simulate the collision cascade process. As a result atomistic simulation methods can play an important role in the understanding of how these glasses behave after a radiation event. Although Molecular Dynamics (MD) simulations can only access very short times $(\sim 1 \mathrm{~ns})$, kinetic Monte Carlo (kMC) simulation techniques can be used to access longer time scales.

In order to simulate the radiation event it is necessary to have a good model of the amorphous glass structure. Amorphous materials are more difficult to handle computationally than crystals since there is no equivalent concept of a vacancy or interstitial. In addition although the structures have short range order, there are many different atomic configurations that are possible so statistical averaging over different structures is very important. In addition good inter-atomic potentials to describe these systems is essential.

In this work we compare three inter-atomic potentials from the literature to test their suitability for molecular dynamics (MD) simulations of radiation effects in borosilicate glass. We investigated two potentials from J. M. Delaye's group (Delaye1996 [1] and Delaye2011 [2]) and one potential from M. Rushton (MRushton2008 [3]). The 'best' potential model was then used to determine threshold displacement energies and some typical energies barriers for atomic movement after such atomic displacements.

\section{Methodology}

Computer simulation of these borosilicate glass systems is performed using MD. A brief overview of each inter-atomic potential that we have investigated is given in the subsections 2.1 to 2.3 below. These potentials are suitable for near equilibrium conditions and must be joined to a screened Coulomb potential for close particle separation. Our splining method is described in section 2.4, and the method for generating glass structures is described in section 2.5.

\subsection{Delaye 1996}

The Delaye1996 potential [1] contains both 2 and 3 body terms. The 2-body interaction is modelled by the Born-Mayer-Huggins potential. The potential energy, $\Phi\left(r_{i j}\right)$, of two ions separated by $r_{i j}$ is given by equation 1 below:

$$
\Phi\left(r_{i j}\right)=A_{i j} \exp \left(\frac{-r_{i j}}{\rho_{i j}}\right)+\frac{1}{4 \pi \epsilon_{0}} \frac{z_{i} z_{j}}{r_{i j}}
$$

where $A_{i j}$, and $\rho_{i j}$ are parameters of the model and can be found in the original paper [1]. $z_{i}$ and $z_{j}$ are the ion charges. This potential uses full formal charges for each ion. 
A Stillinger-Weber [6] 3-body term is applied to the O-Si-O and $\mathrm{Si}-\mathrm{O}-\mathrm{Si}$ bond angles. We choose not to put any restriction on the O-B-O bond angle as was done by Connelly [7]. The adjustable parameters are listed in the original paper [1] in table 4.

Due to the inclusion of the 3-body terms, this potential is the most computationally intensive. The three body term also induces an extremely large penalty when the bond angles move away from their equilibrium values.

\subsection{Delaye2011}

The Delaye2011 potential [2] contains only 2-body terms. A Buckingham pair potential (given by equation 2 below) is used to model these interactions.

$$
\Phi\left(r_{i j}\right)=A_{i j} \exp \left(\frac{-r_{i j}}{\rho_{i j}}\right)+\frac{1}{4 \pi \epsilon_{0}} \frac{z_{i} z_{j}}{r_{i j}}-\frac{C_{i j}}{r_{i j}^{6}}
$$

The adjustable parameters $A_{i j}, \rho_{i j}$ and $C_{i j}$ for each ion interaction are listed in the original paper [2]. The ion charges $\left(z_{i}\right.$ and $\left.z_{j}\right)$, depend upon the glass composition. This composition is characterised by the two molar ratios, $\mathrm{R}=\left[\mathrm{Na}_{2} \mathrm{O}\right] /\left[\mathrm{B}_{2} \mathrm{O}_{3}\right]$ and $\mathrm{K}=\left[\mathrm{SiO}_{2}\right] /\left[\mathrm{B}_{2} \mathrm{O}_{3}\right]$.

For borosilicate glass, $\mathrm{R}=0$, and therefore the charges obtained are close to the values used in the Guillot-Sator potential model [8]: $z_{S i}=1.89, z_{O}=0.945$ and $z_{B}=1.4175$. We used these charges in this work.

The boron-oxygen interaction is a special case. Here the $A_{i j}$ parameter depends on the composition of the glass. For borosilicate glass, $A_{i j}=180390.53 \mathrm{eV}$ and for the boron-oxygen interaction we used, $\rho_{i j}=0.124 \AA$ And $C_{i j}=35.0019 \mathrm{eV} \cdot \AA^{6}$.

The paper also describes inter-atomic potentials for sodium borosilicate glasses. Therefore, we also looked at the SBN12 blend $\left(59.66 \mathrm{SiO}_{2}: 28.14\right.$ $\mathrm{B}_{2} \mathrm{O}_{3}: 12.20 \mathrm{Na}_{2} \mathrm{O}$ ) which we denote as Delaye2011SBN12 in this work. In this case, $\mathrm{R}=0.4335$ and therefore $A_{i j}=205751.1464 \mathrm{eV}$ for the boron-oxygen bond. The ion charges were: $z_{S i}=1.869128, z_{O}=-0.965872, z_{B}=1.528756$ and $z_{N a}=0.451628$. All the remaining parameters are the same as before.

\subsection{MRushton2008}

The MRushton2008 potential [3] also contains only 2-body potentials. In this case, a Lennard-Jones pair potential plus the Coulomb term (equation 3) is used to model the atomic interactions.

$$
\Phi\left(r_{i j}\right)=\frac{\sqrt{A_{i} A_{j}}}{r_{i j}^{12}}-\frac{\sqrt{B_{i} B_{j}}}{r_{i j}^{6}}+\frac{1}{4 \pi \epsilon_{0}} \frac{z_{i} z_{j}}{r_{i j}}
$$

The parameters $A$ and $B$ for each ion are given in the original paper. $z_{i}$ and $z_{j}$ are the full formal charges for each ion. 


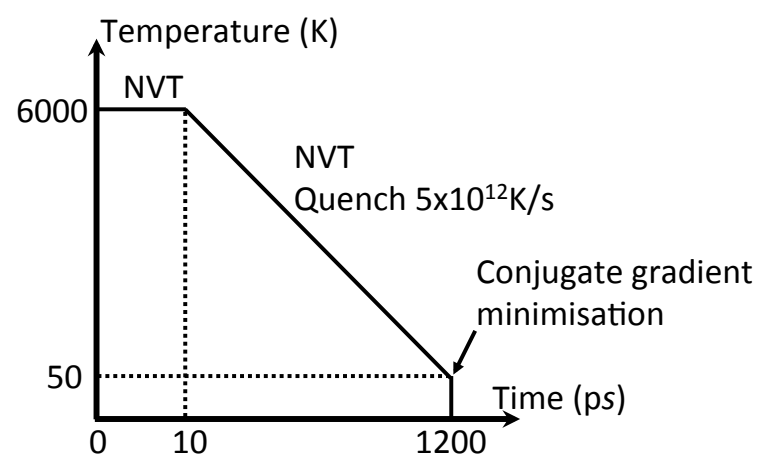

Figure 1: A schematic of the quenching procedure.

\subsection{Application to collision cascades}

Some of these potentials tend to $-\infty$ at short range due to the electrostatic coulomb term $\left(z_{i} z_{j} / r_{i j}\right)$ and the Buckingham term $\left(-C_{i j} / r_{i j}^{6}\right)$. Therefore, the potential energy reaches a maximum value at short range, which can be as low as $150 \mathrm{eV}$ (for a Si-O bond). Hence, during a radiation damage cascade, it becomes possible for some atoms to unnaturally fuse together. The short range interactions between atomic nuclei are more accurately modelled by the ZBL potential [9]. We therefore join these potentials to the ZBL potential at short range with a spline function given by equation 4 .

$$
F=\exp \left(a_{0}+a_{1} r+a_{2} r^{2}+a_{3} r^{3}+a_{4} r^{4}+a_{5} r^{5}\right)
$$

The constants $a_{0}-a_{5}$ are set such that the potential energy and its first and second derivatives are continuous.

\subsection{Modelling of glass formation}

The glass structures are amorphous and have no long range order, therefore we cannot simply generate a structure as we would for a crystal lattice. The procedure for generating a glass structure is to perform an MD simulation of a quench from a molten state.

Atoms are placed at random positions inside a cubic box, ensuring no two atoms are closer than $1 \AA$. Such a configuration has a large potential energy, and therefore a high initial temperature when simulated with MD, typically exceeding $10000 \mathrm{~K}$. At every time-step the temperature is controlled by rescaling the velocities of all the atoms. If the temperature exceeds the desired value by $7 \%$, then the velocities are rescaled to the desired temperature. We equilibrate at $6000 \mathrm{~K}$ for $10 \mathrm{ps}$ then quench at $5 \times 10^{12} \mathrm{~K} / \mathrm{s}$ to $50 \mathrm{~K}$. The final minimisation to $0 \mathrm{~K}$ is obtained via conjugate gradient minimisation. A schematic of this process is shown in figure 1. 


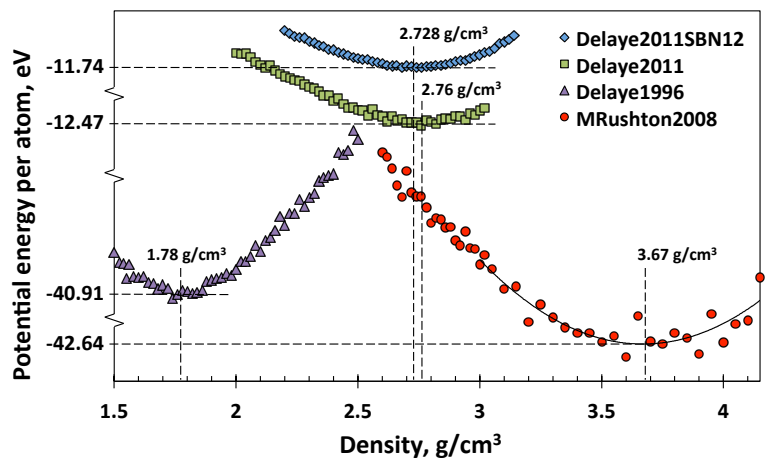

Figure 2: Plots of the average total potential energy per atom of quenched lattices vs density for each inter-atomic potential. Each curve has been offset on the vertical axis so that they appear within one plot.

\section{Results}

\subsection{Density}

For each inter-atomic potential we performed a set of quenches using constant volume MD. We vary the volume to obtain sets quenched glass structures at a range of different densities. Figure 2 shows the plots of the average potential energy per atom as a function of glass density for each inter-atomic potential that we studied. Each plot has been offset on the vertical axis such that all curves appear within the same figure.

In each case, the data about the minimum has been fitted with a $2^{\text {nd }}$ order polynomial, from which we estimate the global minimum lattice energy. We find that the Delaye1996 potential has a global minimum at a density of $1.78 \mathrm{gcm}^{3}$. This is lower than the experimental value of $2.04 \mathrm{gcm}^{3}$ [10]. All the other potentials have global minima that are higher than the experimental values. The Delaye2011 and Delaye2011SBN12 potentials have minima at 2.76 and $2.728 \mathrm{gcm}^{3}$ respectively, and the MRushton2008 potential was higher still at $3.67 \mathrm{gcm}^{3}$.

The MRushton2008 potential produced a glass structure that contained many over co-ordinated silicon and boron atoms. This is the reason for the significantly higher optimum density found.

The higher densities that we found using the Delaye2011 potential could be a high pressure phase that our constant volume simulations are unable to detect. The original authors generated their glass structures by quenching at a density $5-10 \%$ below the experimental value, and then relaxed the system at constant pressure. This enabled them to find a stable local minimum close to the experimental density. In our simulations, a constant pressure minimisation of the optimum glass structures does not significantly change the system volume.

The value of the average potential energy per atom of the optimum glass structures also varies between each potential. 
Table 1: Summary of simulated glass structural properties for each inter-atomic potential

\begin{tabular}{|l|l|l|l|l|l|l|}
\hline Potential & $\begin{array}{l}\text { Density } \\
\left(\mathrm{g} / \mathrm{cm}^{3}\right)\end{array}$ & $K_{B}$ & \multicolumn{2}{|c|}{ Bond length $(\AA)$} & \multicolumn{2}{c|}{ Bond angle $\left(^{\circ}\right)$} \\
\cline { 4 - 7 } & 1.78 & 90 & Si-O & B-O & O-Si-O & O-B-O \\
\hline Delaye1996 & 2.76 & 79 & 1.695 & 1.355 & 109.43 & 119.26 \\
Delaye2011 & 2.728 & 79 & 1.608 & 1.428 & 109.36 & 119.10 \\
Delaye2011SBN12 & 79.34 & 112.93 \\
MRushton & 3.67 & - & 1.93 & 1.468 & 100.89 & 103.10 \\
\hline
\end{tabular}

\subsection{Structural properties}

We measured some structural properties of each glass. This data is summarised in table 1. All of the Delaye potentials give bond length and bond angle distributions that are in good agreement with experimental data. The MRushton2008 potential, however, contains Si-O and B-O bond lengths that are too long. The bond angle distributions in this case also differ due to the over-coordinated structure.

The Bulk moduli, $K_{B}$, of the optimum quenched glass structures for each potential is found using GULP [11], and are reported in table 1. We see that in all cases the bulk moduli for borosilicate glass exceeds the experimental value of 23.7 GPa [2]. The sodium borosilicate glass (modelled using Delaye2011SBN12) structure also has a bulk modulus that exceeds the experimental value of $42 \mathrm{GPa}$. We found that the bulk modulus decreases with decreasing density, and that when quenching a glass using the original authors method, we were able to replicate the results in the original paper, which were much closer to the experimental values.

\subsection{Melting points}

We also measured the melting temperature predicted by each inter-atomic potential. The method for estimating the melting point is to look at the peak of a plot of the specific heat capacity as a function of temperature. Figure 3 shows this for each inter-atomic potential that we investigated. To generate this plot:

1. For a range of temperatures $(0-14000 \mathrm{~K})$, using the optimum quenched lattice for each potential, we thermalise the system using the Berendsen thermostat for $10 \mathrm{ps}$.

2. Then we run a constant energy MD (NVE) simulation for each case, and compute the time average of the temperature and total lattice energy.

3. The specific heat capacity is then the gradient of a plot of the total lattice energy vs temperature.

4. To obtain a smooth curve, we use a least squares fit on several adjacent points to obtain a moving average of the gradient.

We see that the Delaye1996 potential predicts the highest melting point at approximately $8000 \mathrm{~K}$. The MRushton2008 potential predicts the melting point at about $6000 \mathrm{~K}$, and both the Delaye2011 and Delaye2011SBN12 potentials give $2700 \mathrm{~K}$. The experimental value of the melting temperature of sodium borosilicate glass ranges from 1310 to $1870 \mathrm{~K}$ depending upon the composition of the glass $[10,12])$. 


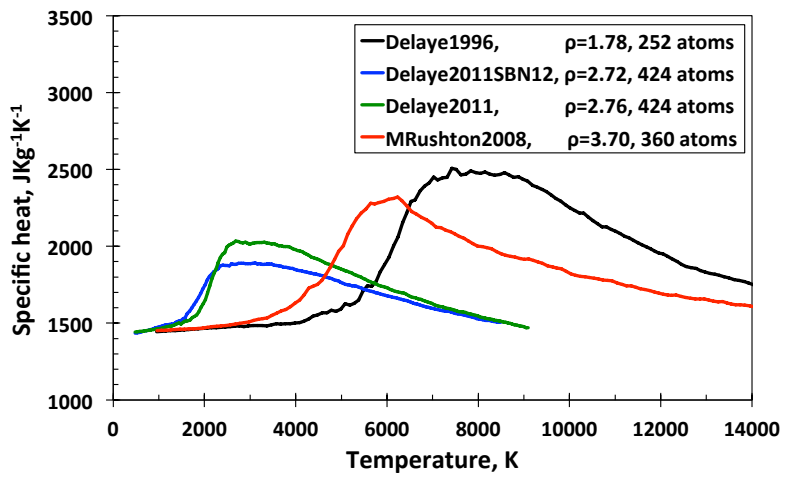

Figure 3: A Plot of the temperature dependence of the specific heat capacity. The peak occurs at 8000 K for Delaye1996, 6000 K for MRushton2008 and 2700 K for Delaye2011 potential.

\subsection{Displacement energy thresholds}

We calculate the displacement threshold energies for each atom species in quenched lattices using the Delaye2011 potential. The procedure we used is:

1. For each atom species, we choose an atom from the quenched lattice at random and pick a random direction in $3 \mathrm{D}$ space.

2. We run an initial trial cascade by adding $100 \mathrm{eV}$ of kinetic energy to the selected atom in the random direction we picked earlier.

3. After 1 ps we compare the initial and final lattices to see if the atom has become permanently displaced. The cut-off for displacement is $1 \AA$.

4. If it has not been displaced, we double the trial energy and rerun the cascade.

5. We find the minimum threshold energy required to permanently displace the atom using the binary search algorithm. We compute the threshold to an accuracy of $1 \mathrm{eV}$ in each case.

The distributions of threshold energies are shown in figure 4 . Here we plot data for borosilicate glass at two densities $\left(2.0\right.$ and $\left.2.76 \mathrm{~g} / \mathrm{cm}^{3}\right)$ modelled with the Delaye2011 potential and sodium borosilicate glass (at the optimum density $2.74 \mathrm{~g} / \mathrm{cm}^{3}$ ) modelled by Delaye2011SBN12 potential.

The average threshold energy does not have any directional dependence upon cascade direction, since the glass structures are amorphous. We also find that the lower density borosilicate glass has lower threshold energies for all atomic species when compared to the higher density case. Compared to ionic crystalline systems, the thresholds are lower [13] with a more continuous spectrum. The systems were also examined after a displacement event. It was found that there were no debonded atoms or voids formed in the system, a result confirmed also by running cascades up to $1 \mathrm{keV}$. Defective regions resulting from these low energy displacements were best identified through the co-ordination number of each atom in the system. 


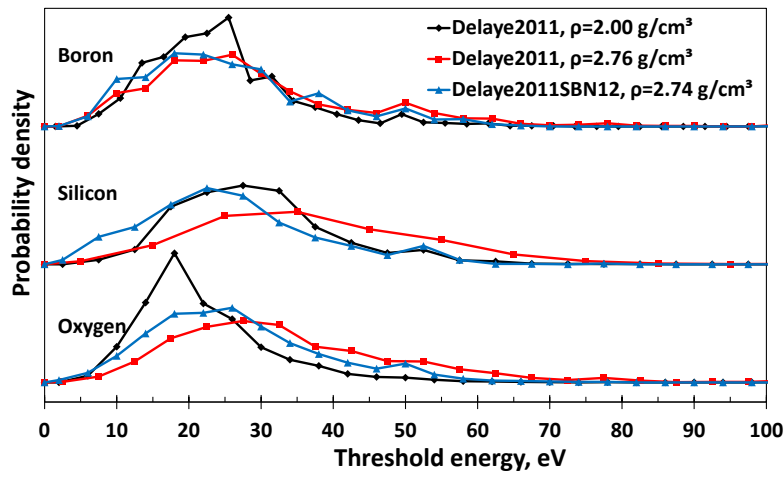

\begin{tabular}{|l|l|l|l|}
\hline \multirow{2}{*}{ Glass } & \multicolumn{3}{|c|}{ Average threshold energy $(\mathrm{eV})$} \\
\cline { 2 - 4 } & Boron & Silicon & Oxygen \\
\hline Delaye2011, $\rho=2.00 \mathrm{~g} / \mathrm{cm}^{3}$ & 24 & 29 & 29 \\
Delaye2011, $\rho=2.76 \mathrm{~g} / \mathrm{cm}^{3}$ & 29 & 37 & 34 \\
Delaye2011SBN12, $\rho=2.74 \mathrm{~g} / \mathrm{cm}^{3}$ & 26 & 26 & 27 \\
\hline
\end{tabular}

Figure 4: The plot shows the displacement energy thresholds for each atomic species (B, Si and $\mathrm{O}$ ) for 3 quenched glass structures. The average threshold energy for each species for each glass is summarised in the above table.

\subsection{Transition barriers}

In an ionic crystal one can determine the activation energies of defects such as vacancies and interstitials in a straightforward way. However, here there is no equivalent concept. Because rebonding occurred after displacement, we determined the longer time scale transition in two ways. In the first way, under co-ordinated atoms were identified and their motion to a higher co-ordinated state was examined. Such motion generally resulted in large energy barriers of $3 \mathrm{eV}$ or more. At $520 \mathrm{~K}$, which is the initial storage temperature of a waste glass due to self-heating from $\beta$-decay [5], a $3 \mathrm{eV}$ barrier is equivalent to one event every 356 million years. So we adopted a different approach. Here we use the data we obtained from threshold calculations above to identify initial and final states of transitions within the glass structures. We use the nudged elastic band method [14] to compute the minimum energy pathway between these states. This gives an estimate of the barrier height for these transitions. Figure 5 shows histograms of barriers found for two glass structures.

For borosilicate glass, the minimum barrier found was $0.9 \mathrm{eV}(52 \mu$ s per event at $520 \mathrm{~K})$. For sodium borosilicate glass, there were more small barriers, however, these only involved movements of $\mathrm{Na}$ atoms only. The minimum barrier found for Si movements in this case was $0.76 \mathrm{eV}(2.3 \mu$ s per event $)$, and $\mathrm{B}$ movements, $0.67 \mathrm{eV}(0.3 \mu$ s per event $)$.

\section{Conclusion}

We have found that all the inter-atomic potentials that we studied predict a melting point that exceeds the experimental value. The most recent potential 
A. Delaye2011

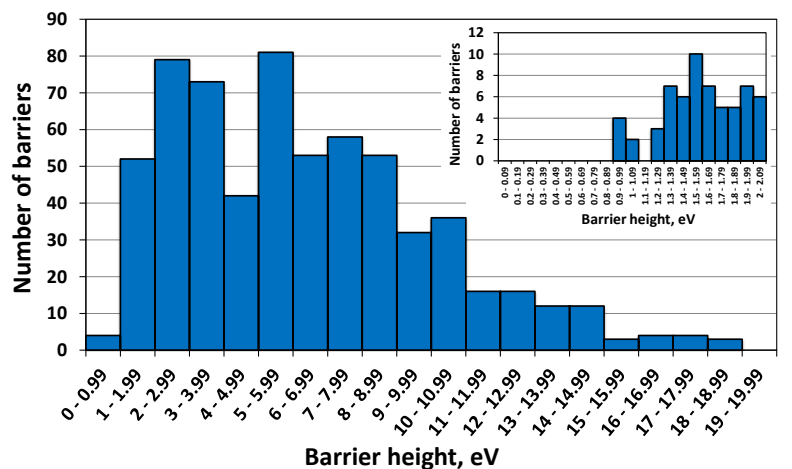

B. Delaye2011SBN12

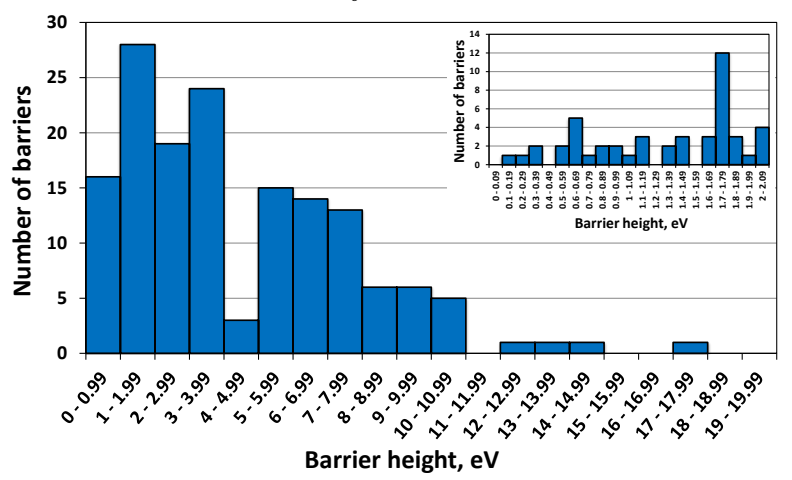

Figure 5: Histogram of transition barriers for (A) borosilicate glass modelled by the Delaye2011 potential and (B) sodium borosilicate glass modelled by Delaye2011SBN12 potential. The insets in each figure show a zoomed in section of the data in the range $0-2 \mathrm{eV}$. 
(Delaye2011) was the closest. We therefore conclude that the best existing interatomic potential for borosilicate glass is the Delaye2011 potential. However, the minimum energy structure has a density that is too high at $\rho=2.76 \mathrm{~g} / \mathrm{cm}^{3}$. It is possible to quench a glass at the experimental density using the original authors method. However in the thermal spike induced by a collision cascade there will be local melting and therefore a likely transition to a higher density state. It is easier for atoms to be displaced in the amorphous glass structures, compared to other ion systems such as spinel [15] by a radiation event but the resulting damaged system is quickly rebonded and does not result in fast diffusion of atoms. This indicates the possibility of modelling multiple radiation events within a system without the necessity to perform kinetic Monte Carlo simulations of diffusion and recombination between radiation events as is required for crystalline materials [16].

\section{Acknowledgements}

We thank Dr J.G Shah of the Bhabia Atomic Research Centre (BARC) and Dr Karl Travis of Sheffield University for useful discussions. Computing resources were provided by Loughborough University HPC service. The work was funded as part of a joint UK-India Nuclear Collaboration through EPSRC grant no. EP/K007882/1

\section{References}

[1] J.-M. Delaye, D. Ghaleb, Molecular dynamics simulation of $\mathrm{SiO}_{2}+\mathrm{B}_{2} \mathrm{O}_{3}$ $+\mathrm{Na}_{2} \mathrm{O}+\mathrm{ZrO}_{2}$ glass, J. Non-Cryst. Solids 195 (3) (1996) 239-248. http://dx.doi.org/10.1016/0022-3093(95)00527-7.

[2] L.-H. Kieu, J.-M. Delaye, L. Cormier, C. Stolz, Development of empirical potentials for sodium borosilicate glass systems, J. Non-Cryst. Solids $357 \quad$ (18) (2011) 3313-3321. http://dx.doi.org/10.1016/j.jnoncrysol.2011.05.024.

[3] M.J.D. Rushton, R.W. Grimes, S.L. Owens, Predicted changes to alkali concentration adjacent to glass-crystal interfaces, J. Am. Ceram. Soc. 91 (5) (2008) 1659-1664. http://dx.doi.org/10.1111/j.1551-2916.2008.02352.x.

[4] J.A.C. Marples, The preparation, properties, and disposal of vitrified high level waste from nuclear fuel reprocessing, Glass technology 29 (6) (1988) 230-247.

[5] W.J. Weber, R.C. Ewing, C.A. Angell, G.W. Arnold, A.N. Cormack, J.-M Delaye, D.L. Griscom, L.W. Hobbs, A. Navrotsky, D.L. Price, A.M. Stoneham, M.C. Weinberg, Radiation effects in glasses used for immobilization of high-level waste and plutonium disposition, J. Mater. Res. 12 (8) (1997) 1946-1978. http://dx.doi.org/http://dx.doi.org/10.1557/JMR.1997.0266. 
[6] F.H. Stillinger, T.A. Weber, Computer simulation of local order in condensed phases of silicon, Phys. Rev. B 31 (8) (1985) 5262-5271. http://dx.doi.org/10.1103/PhysRevB.31.5262.

[7] A.J. Connelly, K.P. Travis, R.J. Hand, N.C. Hyatt, E. Maddrell, Composition-Structure Relationships in Simplified Nuclear Waste Glasses: 1. Mixed Alkali Borosilicate Glasses, J. Am. Ceram. Soc. 94 (1) (2011) 151-159. http://dx.doi.org/10.1111/j.1551-2916.2010.04066.x.

[8] B. Guillot, N. Sator, A computer simulation study of natural silicate melts. Part I: Low pressure properties, Geochim. Cosmochim. Acta 71 (5) (2007) 1249-1265. http://dx.doi.org/10.1016/j.gca.2006.11.015.

[9] J.F. Ziegler, J.P. Biersack, U. Littmark, The Stopping and Range of Ions in Matter, Vol. 1, Pergamon, New York, 1985.

[10] C. Maynell, G. Saunders, S. Scholes, Ultrasound propagation in glasses in the metastable immiscibility region of the sodium borosilicate system, J. Non-Cryst. Solids 12 (3) (1973) 271-294. http://dx.doi.org/10.1016/00223093(73)90001-X.

[11] J.D. Gale, A.L. Rohl, The General Utility Lattice Program ( GULP ), Mol. Simul. 29 (5) (2003) 291-341. http://dx.doi.org/10.1080/0892702031000104887.

[12] R.L. Hervig, A. Navrotsky, Thermochemistry of Sodium Borosilicate Glasses, J. Am. Ceram. Soc. 68 (6) (1985) 314-319. http://dx.doi.org/10.1111/j.1151-2916.1985.tb15232.x.

[13] L. Kittiratanawasin, R. Smith, B. Uberuaga, K. Sickafus, Displacement threshold and Frenkel pair formation energy in ionic systems, Nucl. Instr. Meth. B 268 (19) (2010) 2901-2906. http://dx.doi.org/10.1016/j.nimb.2010.04.024.

[14] G. Henkelman, B.P. Uberuaga, H. Jónsson, A climbing image nudged elastic band method for finding saddle points and minimum energy paths, J. Chem. Phys. 113 (22) (2000) 9901. http://dx.doi.org/10.1063/1.1329672.

[15] R. Smith, D. Bacorisen, B.P. Uberuaga, K.E. Sickafus, J.A. Ball, R.W. Grimes, Dynamical simulations of radiation damage in magnesium aluminate spinel, $\mathrm{MgAl}_{2} \mathrm{O}_{4}$, J. Phys.: Condens. Matter 17 (6) (2005) 875891. http://dx.doi.org/10.1088/0953-8984/17/6/008.

[16] C. Scott, R. Smith, Modelling the sputtering of Au surfaces using a multi time-scale technique, Proc. R. Soc. A 469 (2150) (2012) 20120480. http://dx.doi.org/10.1098/rspa.2012.0480. 